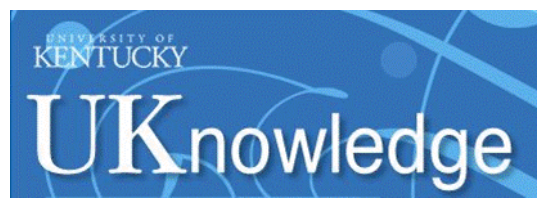

University of Kentucky

UKnowledge

3-10-2005

\title{
Theoretical He I Emissivities in the Case B Approximation
}

\author{
R. L. Porter \\ University of Kentucky, rporter@pa.uky.edu \\ R. P. Bauman \\ University of Kentucky \\ Gary J. Ferland \\ University of Kentucky, gary@uky.edu \\ K. B. MacAdam \\ University of Kentucky
}

Follow this and additional works at: https://uknowledge.uky.edu/physastron_facpub

Part of the Astrophysics and Astronomy Commons, and the Physics Commons

Right click to open a feedback form in a new tab to let us know how this document benefits you.

\section{Repository Citation}

Porter, R. L.; Bauman, R. P.; Ferland, Gary J.; and MacAdam, K. B., "Theoretical He I Emissivities in the Case B Approximation" (2005). Physics and Astronomy Faculty Publications. 94.

https://uknowledge.uky.edu/physastron_facpub/94

This Article is brought to you for free and open access by the Physics and Astronomy at UKnowledge. It has been accepted for inclusion in Physics and Astronomy Faculty Publications by an authorized administrator of UKnowledge. For more information, please contact UKnowledge@lsv.uky.edu. 


\section{Theoretical He I Emissivities in the Case B Approximation}

Digital Object Identifier (DOI)

http://dx.doi.org/10.1086/429370

\section{Notes/Citation Information}

Published in The Astrophysical Journal Letters, v. 622, no. 1, p. L73-L75.

(C) 2005. The American Astronomical Society. All rights reserved. Printed in the U.S.A.

The copyright holder has granted permission for posting the article here. 


\title{
THEORETICAL He I EMISSIVITIES IN THE CASE B APPROXIMATION
}

\author{
R. L. Porter, R. P. Bauman, G. J. Ferland, and K. B. MacAdam \\ Department of Physics and Astronomy, University of Kentucky, 177 Chemistry/Physics Building, Lexington, KY 40506; rporter@ pa.uky.edu \\ Received 2004 October 22; accepted 2005 February 3; published 2005 February 28
}

\begin{abstract}
We calculate the He I case B recombination cascade spectrum using improved radiative and collisional data. We present new emissivities over a range of electron temperatures and densities. The differences between our results and the current standard are large enough to have a significant effect not only on the interpretation of observed spectra of a wide variety of objects, but also on determinations of the primordial helium abundance.
\end{abstract}

Subject headings: atomic data — atomic processes - ISM: atoms - ISM: clouds — plasmas

\section{INTRODUCTION}

Helium is the second most abundant element in the universe, and its emission and opacity help us determine the structure of any interstellar cloud. Its abundance relative to hydrogen can be measured within a few percent since the emissivities of $\mathrm{H} \mathrm{I}$ and $\mathrm{He} \mathrm{I}$ lines have similar dependences on temperature and density. This makes it an indicator of both stellar and primordial nucleosynthesis (Pagel 1997).

A good discussion of the history of calculations of the helium recombination spectra is given by Benjamin et al. (1999, hereafter B99), who present new calculations - the current standard in the field. Yet much progress has been made since the work by Smits $(1991,1996)$ on which the B99 results depend. We implement these improvements, present a new set of predictions, and compare our results with those of B99. The differences are large enough to impact continuing attempts to estimate the primordial helium abundance (Peimbert et al. 2002).

\section{THE NEW MODEL HELIUM ATOM}

The basic physical processes have been described by Brocklehurst (1972) and B99. Here we describe the differences between B99 and our new numerical representation of the helium atom, which is a part of the spectral simulation code CLOUDY (Ferland et al. 1998). This model resolves all terms, $n l S$, up to an adjustable maximum principal quantum number $n_{\max }$, followed by a pseudolevel, $n_{\max }+1$, in which all $l S$ terms are assumed to be populated according to statistical weight and "collapsed" into one. We set recombinations into the collapsed level equal to the convergent sum of recombinations from $n=n_{\max }+1$ to $\infty$. In the low-density limit, the collapsed level increases the emissivities of our benchmark lines (the same 32 lines given in B99) by $0.4 \%$, on average, with $n_{\max }=100$. The decays from states with $l=n-1$ are most sensitive to this correction for system truncation. The strong optical line $\lambda 5876$ is corrected upward by $1.3 \%$. At finite densities collisional processes force the populations of very highly excited states into local thermodynamic equilibrium (LTE). In this case the adequacy of the method used to compensate for truncation is unimportant. We find the corrections negligible for $n_{e}=100 \mathrm{~cm}^{-3}$ and $n_{\max }=100$. Consequently, the uncertainties in the results presented in $\S 3$ are due to the uncertainties in atomic data, especially the often substantial uncertainties in collisional rates affecting terms not in LTE at given conditions.

There are several differences in atomic data for radiative processes between B99 and the present work. The transition probabilities and radiative recombination coefficients are ob- tained from oscillator strengths and photoionization cross sections. B99 uses the oscillator strengths calculated by Kono \& Hattori (1984). While these agree very well with the essentially exact oscillator strengths of Drake (1996), ${ }^{1}$ Drake presents a much larger set, up to and including $n=10$ and $l=7$, which we adopt. Hummer \& Storey (1998, hereafter HS98) have presented ab initio calculations of threshold photoionization cross sections up to $n=4$. B99 uses cross sections from TOPbase ${ }^{2}$ (Cunto et al. 1993), while we use the more accurate HS98 values. The dominant remaining uncertainties in radiative data are in oscillator strengths involving low $l$ states (with $n>10$ ) and photoionization cross sections for low $l$ states (with $n>$ 4). HS98 also illustrate the method, originally discussed by Seaton (1958), of calculating threshold photoionization cross sections by extrapolating absorption oscillator strengths to the threshold energy of a given level. This method has been used in the present work, based on the oscillator strengths from Drake, to extend the ab initio cross sections of HS98 to greater $n$.

Differences in collisional data between B99 and the current work are also significant. For low- $n$ transitions for which there are ab initio calculations, B99 uses the collision strengths of Sawey \& Berrington (1993). We replace these, where available, with the results of the close-coupling calculation by Bray et al. (2000), which include continuum states not considered in the $R$-matrix calculations by Sawey \& Berrington.

For $l$-changing collisions, B99 use two different treatments: Seaton (1962, hereafter S62) for low- $l$ transitions and Pengelly \& Seaton (1964, hereafter PS64) otherwise. Neither of these treatments allows for angular momentum transfers greater than one unit, and both apply when the projectile velocity is greater than the velocity of the bound electron. The rms electron and projectile velocities in conditions considered by B99, assuming proton colliders, are

$$
v_{e}=\frac{Z \alpha c}{n} \text { and } v_{\mathrm{proj}}=\sqrt{\frac{3 k T}{m_{p}}}
$$

where $\alpha$ is the fine structure constant, $c$ is the speed of light, and $Z$ is the screened nuclear charge. Dividing the latter by the former, we arrive at the expected value of the reduced

\footnotetext{
${ }^{1}$ Oscillator strengths taken from extended tables received via 2002 private communication from G. W. F. Drake.

${ }^{2}$ See http://vizier.u-strasbg.fr/topbase/topbase.html.
} 


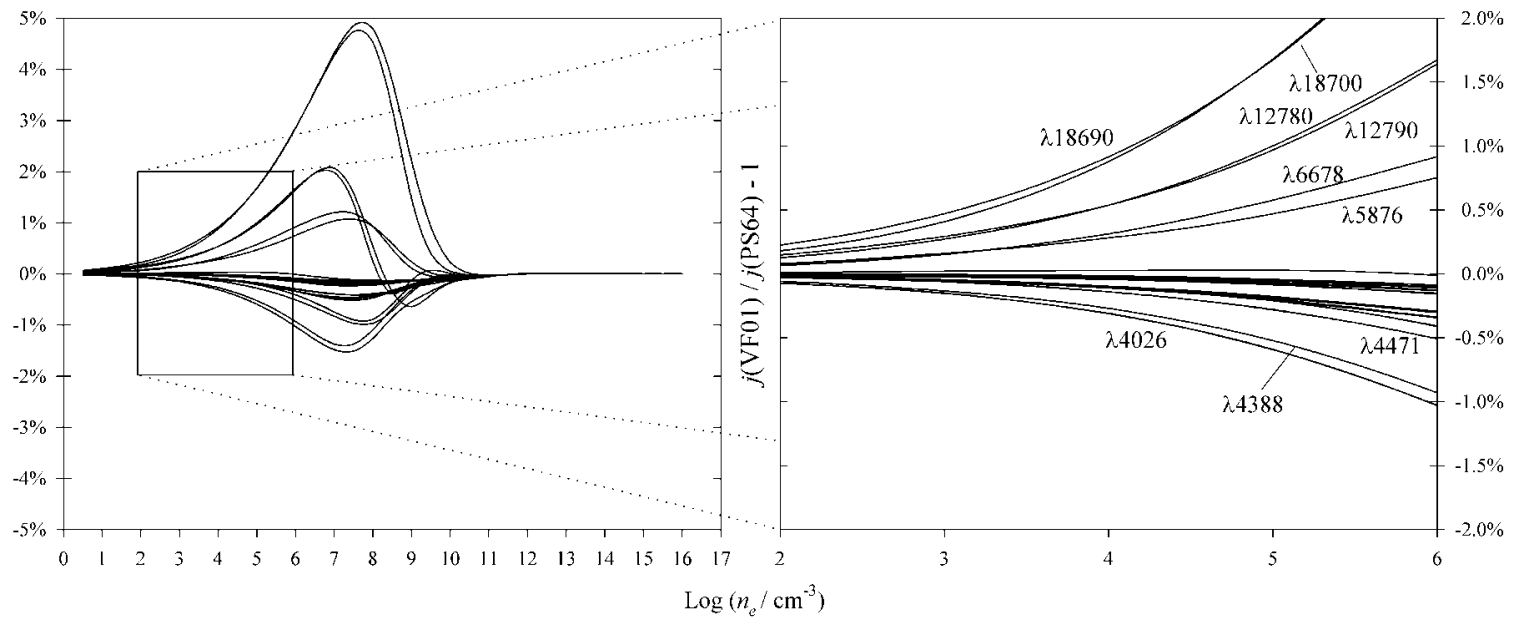

FIG. 1.-Percent difference between the emissivities calculated using two different Stark collision treatments, for several strong lines, as a function of $n_{e}$. Left: Wide range of densities - as expected, there is no effect in either the low-density extreme, because the collision rates are negligible, or the high-density extreme, where the Stark collisions force the terms to LTE. The majority of lines are most sensitive at densities found in stellar envelopes and quasar emissionline regions. Right: Range of densities found in nebulae-several lines have a sensitivity to the Stark collision treatment of about $1 \%$.

velocity as a function of temperature and principal quantum number

$$
\langle\tilde{v}\rangle=\frac{v_{\text {proj }}}{v_{e}}=7.19 \times 10^{-5} n \sqrt{T(\mathrm{~K})} .
$$

For typical nebular temperatures, this reduced velocity will be of the order or less than unity for proton colliders for all $n \leq 150$; the treatment of PS64 is applicable only for greater $n$, and it greatly overestimates $l$-mixing cross sections when used outside its range of validity (MacAdam et al. 1981).

Vrinceanu \& Flannery (2001, hereafter VF01) give a (classical) theory of $l$-changing collisions and claim exact solutions in the limit that the intrashell transition is induced by slow distant collisions. Their treatment allows naturally for angular momentum changes greater than unity. (At a sufficiently high reduced velocity, large angular momentum transfers are strongly suppressed and the theory goes to the optically allowed limit with which PS64 are concerned.) We use equation (41) of Kazansky \& Ostrovsky (1996) for the angle, $\Delta \Phi$, swept out by the projectile. A physical basis for the necessary large impact parameter cutoff in the theory follows from equating the

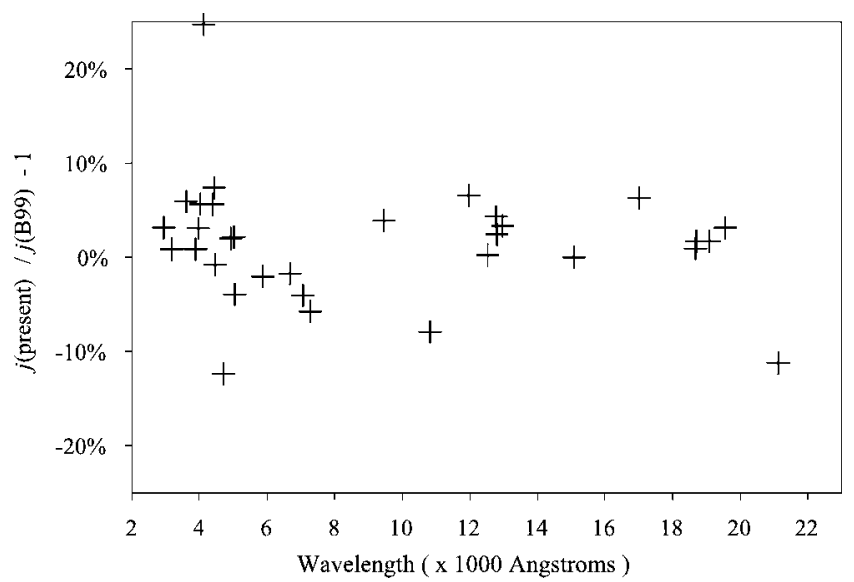

FIG. 2.-Comparison of the present results with those of B99 at $T_{e}=10^{4} \mathrm{~K}$ and $n_{e}=10^{4} \mathrm{~cm}^{-3}$.
Stark and quantum-defect precession frequencies. The Stark frequency is given by

$$
\omega_{s}=\frac{3 Z_{1} n}{2 b^{2}} \text { a.u., }
$$

and the quantum-defect precession frequency (Hezel et al. 1992) is given by

$$
\omega_{\mathrm{qd}}=\frac{5 \delta_{l}}{n^{3} l}\left(1-\frac{3 l^{2}}{5 n^{2}}\right) \text { a.u., }
$$

where $\delta_{l}$ is the quantum defect, $Z_{1}$ is the charge of the projectile, and $b$ is the impact parameter. By setting $\omega_{\mathrm{qd}}$ equal to $\omega_{s}$, we obtain a maximum impact parameter $b_{\max }$. The electron orbit precession will be faster than the Stark beating at larger impact parameters, so that transitions are increasingly less likely. To insure symmetry, we use the average $\omega_{\mathrm{gd}}$ of the initial and final levels. We use VF01 for $l$-changing collisions involving initial and final levels with $l \geq 3$, and like B99 we use the impact parameter treatment of S62 for $l$-change from $s, p$, and $d$ levels. We use electron, proton, and $\mathrm{He}^{+}$colliders for all transitions, taking $n_{\mathrm{He}^{+}}=0.1 n_{p}$ and $n_{e}=n_{p}+n_{\mathrm{He}^{+}}$. Since S62, which describes electron collisions, is based on the method of virtual quanta (see Jackson 1999), we can readily adapt it for the positive-ion collisions: The power spectrum of the timedependent fields generated at the target atom by a passing charged projectile depends only on the projectile's charge magnitude, speed (not kinetic energy or mass separately), and impact parameter. The same considerations apply to PS64 and VF01 and have been implemented to allow for all three collider species. In calculating the necessary thermal averages we have assumed that the same temperature characterizes electrons, protons, and $\mathrm{He}^{+}$ions.

Figure 1 compares emissivities that we predicted using the VF01 and PS64 theories. The predicted emissivities typically change by about $1 \%$ for nebular densities by using the theory of VF01 rather than that of PS64. The difference is much greater at high densities found, for example, in parts of quasars. 
TABLE 1

He i Case B Emissivities

\begin{tabular}{|c|c|c|c|c|c|c|c|c|c|}
\hline \multirow{2}{*}{$\begin{array}{c}\lambda \\
(\AA)\end{array}$} & \multicolumn{3}{|c|}{$T_{e}=5000 \mathrm{~K}$} & \multicolumn{3}{|c|}{$T_{e}=10,000 \mathrm{~K}$} & \multicolumn{3}{|c|}{$T_{e}=20,000 \mathrm{~K}$} \\
\hline & $n_{e}=10^{2} \mathrm{~cm}^{-3}$ & $n_{e}=10^{4} \mathrm{~cm}^{-3}$ & $n_{e}=10^{6} \mathrm{~cm}^{-3}$ & $n_{e}=10^{2} \mathrm{~cm}^{-3}$ & $n_{e}=10^{4} \mathrm{~cm}^{-3}$ & $n_{e}=10^{6} \mathrm{~cm}^{-3}$ & $n_{e}=10^{2} \mathrm{~cm}^{-3}$ & $n_{e}=10^{4} \mathrm{~cm}^{-3}$ & $n_{e}=10^{6} \mathrm{~cm}^{-3}$ \\
\hline 2945 & 0.4142 & 0.4261 & 0.4567 & 0.2687 & 0.2816 & 0.2958 & 0.1648 & 0.1987 & 0.2112 \\
\hline $3889 \quad \ldots \ldots \ldots$ & 2.2452 & 2.3261 & 2.5038 & 1.4116 & 1.6794 & 1.8348 & 0.8315 & 1.3332 & 1.4859 \\
\hline 3965 & 0.2280 & 0.2353 & 0.2532 & 0.1409 & 0.1471 & 0.1543 & 0.0807 & 0.0966 & 0.1020 \\
\hline 4026 & 0.5279 & 0.5427 & 0.5866 & 0.2917 & 0.3029 & 0.3175 & 0.1457 & 0.1782 & 0.1896 \\
\hline 4438 . & 0.0145 & 0.0149 & 0.0157 & 0.0101 & 0.0111 & 0.0117 & 0.0070 & 0.0100 & 0.0107 \\
\hline $4471 \ldots \ldots \ldots$ & 1.1469 & 1.1781 & 1.2699 & 0.6124 & 0.6465 & 0.6806 & 0.3010 & 0.4077 & 0.4418 \\
\hline $4713 \ldots \ldots$ & 0.0904 & 0.0929 & 0.0977 & 0.0652 & 0.0833 & 0.0917 & 0.0478 & 0.0957 & 0.1087 \\
\hline $4922 \ldots \ldots \ldots$ & 0.3132 & 0.3222 & 0.3463 & 0.1655 & 0.1722 & 0.1801 & 0.0799 & 0.0973 & 0.1027 \\
\hline $5016 \ldots \ldots \ldots$ & 0.5849 & 0.6039 & 0.6498 & 0.3539 & 0.3808 & 0.4035 & 0.1996 & 0.2554 & 0.2735 \\
\hline 5048 & 0.0355 & 0.0366 & 0.0387 & 0.0244 & 0.0281 & 0.0301 & 0.0167 & 0.0253 & 0.0276 \\
\hline 5876 & 3.3613 & 3.4419 & 3.6899 & 1.6344 & 1.8724 & 2.0179 & 0.7887 & 1.4753 & 1.6649 \\
\hline $11,970 \ldots \ldots$ & 0.0532 & 0.0547 & 0.0591 & 0.0294 & 0.0305 & 0.0320 & 0.0146 & 0.0179 & 0.0191 \\
\hline $12,530 \ldots \ldots$ & 0.0278 & 0.0286 & 0.0307 & 0.0179 & 0.0195 & 0.0208 & 0.0109 & 0.0146 & 0.0158 \\
\hline $12,780 \ldots \ldots$ & 0.2010 & 0.2056 & 0.2196 & 0.0936 & 0.0972 & 0.1011 & 0.0410 & 0.0513 & 0.0546 \\
\hline $12,800 \ldots \ldots$ & 0.0670 & 0.0686 & 0.0728 & 0.0311 & 0.0320 & 0.0330 & 0.0137 & 0.0169 & 0.0175 \\
\hline $12,970 \ldots \ldots$ & 0.0178 & 0.0183 & 0.0198 & 0.0097 & 0.0101 & 0.0105 & 0.0048 & 0.0056 & 0.0059 \\
\hline $15,080 \ldots \ldots$ & 0.0121 & 0.0125 & 0.0134 & 0.0074 & 0.0078 & 0.0082 & 0.0042 & 0.0051 & 0.0054 \\
\hline $17,000 \ldots \ldots$ & 0.0811 & 0.0833 & 0.0898 & 0.0433 & 0.0457 & 0.0481 & 0.0212 & 0.0288 & 0.0312 \\
\hline $18,680 \ldots \ldots$ & 0.5062 & 0.5126 & 0.5412 & 0.2184 & 0.2282 & 0.2367 & 0.0955 & 0.1261 & 0.1324 \\
\hline $18,700 \ldots \ldots$ & 0.1687 & 0.1711 & 0.1793 & 0.0727 & 0.0748 & 0.0768 & 0.0321 & 0.0449 & 0.0445 \\
\hline $19,090 \ldots \ldots$ & 0.0289 & 0.0297 & 0.0319 & 0.0152 & 0.0159 & 0.0166 & 0.0073 & 0.0089 & 0.0094 \\
\hline $19,550 \ldots \ldots$ & 0.0162 & 0.0167 & 0.0179 & 0.0104 & 0.0114 & 0.0121 & 0.0064 & 0.0085 & 0.0092 \\
\hline $21,120 \ldots \ldots$ & 0.0138 & 0.0141 & 0.0149 & 0.0099 & 0.0127 & 0.0139 & 0.0073 & 0.0146 & 0.0166 \\
\hline
\end{tabular}

NOTES. - Emissivities $4 \pi j_{\lambda} / n_{e} n_{\mathrm{He}^{+}}$are given in units of $10^{-25} \mathrm{ergs} \mathrm{cm}^{3} \mathrm{~s}^{-1}$. The hydrogen density is $0.9 n_{e}$, and the helium abundance is one-tenth of the hydrogen abundance.

\section{RESULTS}

In Figure 2 we compare our results with those of B99 for the case $T_{e}=10^{4} \mathrm{~K}$ and $n_{e}=10^{4} \mathrm{~cm}^{-3}$. The average difference for the 32 emission lines is $4.6 \%$. The greatest difference is for $\lambda 4121$, for which our emissivity is $25 \%$ greater. In general, agreement worsens with increasing density; at $n_{e}=10^{2} \mathrm{~cm}^{-3}$, the average and greatest differences are $1.6 \%$ and $6.4 \%$, respectively, while at $n_{e}=10^{6} \mathrm{~cm}^{-3}$ we find differences of $7.0 \%$ and $35 \%$. Agreement also worsens with increasing temperature. Table 1 presents emissivities for all of the temperatures and densities considered by B99. We believe that these results are a significant improvement. The application of these results to specific astrophysical problems will be the subject of future papers.

We thank G. W. F. Drake for making available extensive tables of his calculations, D. Vrinceanu and M. J. Cavagnero for helpful discussions, and referee Peter Storey, whose constructive criticisms helped us to significantly improve our work. We also acknowledge support from NASA grant NAG5-12020 and NSF grant AST 03-07720.

\section{REFERENCES}

Benjamin, R. A., Skillman, E. D., \& Smits, D. P. 1999, ApJ, 514, 307 (B99) Bray, I., Burgess, A., Fursa, D. V., \& Tully, J. A. 2000, A\&AS, 146, 481 Brocklehurst, M. 1972, MNRAS, 157, 211

Cunto, W., Mendoza, C., Ochsebein, F., \& Zeippen, C. J. 1993, A\&A, 275, L5

Drake, G. W. F. 1996, in Atomic, Molecular, and Optical Physics Handbook, ed. G. W. F. Drake (Woodbury: AIP), 154

Ferland, G. J., Korista, K. T., Verner, D. A., Ferguson, J. W., Kingdon, J. B., \& Verner, E. M. 1998, PASP, 110, 761

Hezel, T. P., Burkhardt, C. E., Ciocca, M., He, L.-W., \& Leventhal, J. J. 1992, Am. J. Phys., 60, 329

Hummer, D. G., \& Storey, P. J. 1998, MNRAS, 297, 1073 (HS98)

Jackson, J. D. 1999, Classical Electrodynamics (3rd ed.; New York: Wiley)

Kazansky, A. K., \& Ostrovsky, V. N. 1996, J. Phys. B: At. Mol. Opt. Phys., 29,3651
Kono, A., \& Hattori, S. 1984, Phys. Rev. A, 29, 2981

MacAdam, K. B., Rolfes, R., \& Crosby, D. A. 1981, Phys. Rev. A, 24, 1286 Pagel, B. E. J. 1997, Nucleosynthesis and Chemical Evolution of Galaxies (Cambridge: Cambridge Univ. Press)

Peimbert, A., Peimbert, M., \& Luridiana, V. 2002, ApJ, 565, 668

Pengelly, R. M., \& Seaton, M. J. 1964, MNRAS, 127, 165 (PS64)

Sawey, P. M. J., \& Berrington, K. A. 1993, At. Data Nucl. Data Tables, 55, 81

Seaton, M. J. 1958, MNRAS, 118, 504

. 1962, Proc. Phys. Soc., 79, 1105 (S62)

Smits, D. P. 1991, MNRAS, 248, 193

. 1996, MNRAS, 278, 683

Vrinceanu, D., \& Flannery, M. R. 2001, Phys. Rev. A, 63, 032701 (VF01) 